\title{
Perturbed Floer homology of some fibered three-manifolds
}

\author{
ZHONGTAO WU
}

In this paper, we write down a special Heegaard diagram for a given product threemanifold $\Sigma_{g} \times S^{1}$. We use the diagram to compute the perturbed $\widehat{H F}$ for the 3 -torus and the perturbed $H F^{+}$for nontorsion $\operatorname{spin}^{c}$-structures for $\Sigma_{g} \times S^{1}$ when $g \geq 2$.

53D40

\section{Introduction}

Heegaard Floer homology was introduced by Ozsváth and Szabó $[4 ; 5]$ and has proved to be a powerful 3-manifold invariant. The construction of the invariant requires an admissibility condition though, which in general is not met by those "simplest" Heegaard diagrams for a given 3 -manifold $Y$ with $b_{1}(Y) \geq 1$. A variant of the construction using the Novikov ring overcomes this shortcoming, and in some sense embraces the ordinary homology as a special case. The invariants, usually called perturbed Heegaard Floer homology, have proved to be useful in some situations. For example, Jabuka and Mark made use of them in calculating Ozsváth-Szabó invariants for certain closed 4-manifolds [1].

This paper aims to compute the perturbed Heegaard Floer homologies for product three-manifolds $\Sigma_{g} \times S^{1}$. The result is a little surprising as we find that the homology groups are independent of the exact direction of perturbations.

We would also like to point out that although the computation is made solely for the product three-manifolds in this paper, the method can in fact be applied to the more general setting of certain fibered three-manifolds; see the author's paper [8].

This paper is organized as follows: In Section 2, we review the backgrounds of the Novikov ring $\mathbb{A}$ and the perturbed Heegaard Floer homology. Treating homology groups as $\mathbb{A}$-vector spaces, we prove a rank inequality and an Euler characteristic identity. In Section 3, we write down a special Heegaard diagram for $T^{3}$ and compute its perturbed Heegaard Floer homology. In Section 4, we compute the homology for nontorsion $\operatorname{Spin}^{c}$ structures for $\Sigma_{g} \times S^{1}$. 
Acknowledgments I would like to thank my advisor, Zoltán Szabó, for introducing me to the problem and having many helpful discussions at various points. I am also grateful to Yinghua Ai, Joshua Greene and Yi Ni for conversations about this work.

\section{Preliminaries on perturbed Heegaard Floer homology}

Ozsváth and Szabó [5, Section 11] sketch a variant of Heegaard Floer homologies analogous to the perturbed version of Seiberg-Witten Floer homology. For its construction, we work over the Novikov ring $\mathbb{A}$ (which is in fact a field) consisting of formal power series $\sum_{r \in \mathbb{R}} a_{r} T^{r}$, for which $a_{r} \in \mathbb{Z}_{2}$ and $\#\left\{a_{r} \mid a_{r} \neq 0, r<N\right\}<\infty$ for any $N \in \mathbb{R}$, endowed with the multiplication law

$$
\left(\sum_{r \in \mathbb{R}} a_{r} T^{r}\right) \cdot\left(\sum_{r \in \mathbb{R}} b_{r} T^{r}\right)=\sum_{r \in \mathbb{R}}\left(\sum_{s} a_{s} b_{r-s}\right) T^{r} .
$$

For a pointed Heegaard diagram $(\Sigma, \alpha, \beta, z)$ for $Y$, define the boundary map $\partial$ by

$$
\partial^{+}[x, i]=\sum_{y}\left(\sum_{\left\{\phi \in \pi_{2}(x, y) \mid n_{z}(\phi) \leq i\right\}} \# \widehat{\mathcal{M}}(\phi) T^{\mathcal{A}(\phi)} \cdot\left[y, i-n_{z}(\phi)\right]\right),
$$

where $\mathcal{A}(\phi)$ denotes the area of the domain $\mathcal{D}(\phi)$. This construction depends on the area of each periodic domain, which can be thought of as a real two-dimensional cohomology class $\eta \in H^{2}(Y ; \mathbb{R})$. It is also shown that the corresponding homology groups, denoted by $\mathrm{HF}^{+}(Y, \mathfrak{s} ; \eta)$, are invariants of the underlying topological data only.

It is a natural question to ask for an explicit dependence of $H F^{\circ}(Y ; \eta)$ on $\eta$. We have not achieved this yet, but our result provides a bound for the rank of $\widehat{H F}(Y ; \eta)$ as a vector space over $\mathbb{A}$. More precisely, it is bounded by $\widehat{H F}(Y ; \omega)$ and $\widehat{H F}(Y ; \Omega)$ for two very special cohomology classes $[\omega]$ and $[\Omega]$, where $[\omega]$ is a generic class in the sense that $\omega(\mathcal{D}) \neq 0$ for any integral periodic domain $\mathcal{D}$ and $\Omega$ is a trivial class where $\Omega(\mathcal{D})=0$ for any periodic domain $\mathcal{D}$.

Proposition 2.1 (1) The rank of $\widehat{H F}(Y ; \Omega)$ over $\mathbb{A}$ is the same as the rank of the ordinary unperturbed $\widehat{H F}(Y)$ over $\mathbb{Z}_{2}$.

(2) The rank of $\widehat{H F}(Y ; \omega)$ over $\mathbb{A}$ is the same as the rank of the nontorsion part of the completely twisted $\underline{\widehat{H F}}\left(Y ; \mathbb{Z}_{2}\left[H^{1}(Y ; \mathbb{Z})\right]\right)$ over the ring $\mathbb{Z}\left[H^{1}(Y ; \mathbb{Z})\right]$.

(3) In general, we have a rank inequality

$$
\operatorname{rank} \widehat{H F}(Y ; \omega) \leq \operatorname{rank} \widehat{H F}(Y ; \eta) \leq \operatorname{rank} \widehat{H F}(Y ; \Omega) .
$$


The proof is based on the following simple fact from linear algebra:

Lemma 2.2 The rank of a matrix $M$ is the largest integer $n$ such that there exists some $n \times n$ minor of $M$ with nonzero determinant.

Note that Lemma 2.2 provides us an algorithm to compute the rank of homology: choose a basis for the vector space $\widehat{C F}$, and write the boundary map $\partial$ in a matrix form $M$. By definition, $\widehat{H F}=\operatorname{Ker} M / \operatorname{Im} M$ and $\operatorname{dim}(\operatorname{Ker} M)+\operatorname{dim}(\operatorname{Im} M)=\operatorname{dim} \widehat{C F}$, so

$$
\operatorname{rank} \widehat{H F}=\operatorname{dim} \operatorname{Ker} M-\operatorname{dim} \operatorname{Im} M=\operatorname{dim} \widehat{C F}-2 \operatorname{rank} M .
$$

In other words, in order to find the rank of $\widehat{H F}$, it suffices to find the rank of $M$, which in turn is completely determined by the determinants of all its minors.

Both $\mathbb{A}$ and $\mathbb{Z}_{2}\left[H^{1}(Y ; \mathbb{Z})\right]$ consist of formal power series as their elements - this is a special property we are going to employ in deciding if a determinant is zero. More specifically, for a matrix $\left(M_{i j}\right)=\left(T^{\phi_{i j}}\right) \in \operatorname{Mat}\left(\mathbb{Z}_{2}\left[H^{1}(Y ; \mathbb{Z})\right]\right)$,

$$
\operatorname{det} M=\sum_{\left\{\sigma_{1}, \sigma_{2}, \ldots, \sigma_{n}\right\}=\{1,2, \ldots, n\}} T^{\phi_{1 \sigma_{1}}+\phi_{2 \sigma_{2}}+\cdots+\phi_{n \sigma_{n}}} .
$$

Being a formal sum, terms can not be added unless their exponents are equal. Hence, $\operatorname{det} M=0$ if and only if we can pair all the terms in the summand and paired terms are cancelled out. More formally, we find $n ! / 2$ pairs, where within each pair of permutations $\sigma$ and $\rho$ we have $T^{\phi_{1 \sigma_{1}}+\phi_{2 \sigma_{2}}+\cdots+\phi_{n \sigma_{n}}}=T^{\phi_{1 \rho_{1}}+\phi_{\rho_{2}}+\cdots+\phi_{n \rho_{n}}}$, or equivalently

$$
\phi_{1 \sigma_{1}}+\phi_{2 \sigma_{2}}+\cdots+\phi_{n \sigma_{n}}=\phi_{1 \rho_{1}}+\phi_{\rho_{2}}+\cdots+\phi_{n \rho_{n}} .
$$

In general, entries of $M$ don't have to be monomials like $T^{\phi_{i j}}$; some entries could be like $T^{\phi_{i j}^{1}+\phi_{i j}^{2}+\cdots}$ and some may vanish. These conditions may occur either when there are more than two holomorphic disks connecting two generators, or there is no disk. Nonetheless, we are still able to write the determinants as sums of the products of entries, and whether det $=0$ or not is still dependent on the existence of the aforementioned pairing. While finding the exact pairing could be difficult, we will only apply the following simple philosophy: "the more terms in the summand are equal, the more likely the sum is zero." This philosophy is only valid in those fields with characteristic 2 and whose elements are formal sums. Fortunately, that is so for $\mathbb{A}$ and $\mathbb{Z}_{2}\left[H^{1}(Y ; \mathbb{Z})\right]$.

Proof of Proposition 2.1 Fix an admissible diagram for $Y$, and find all generators $x_{i} \in \widehat{C F}(Y)$. If the boundary map is given by

$$
\partial x_{i}=\sum_{j}\left(\sum_{\phi \in \pi_{2}\left(x_{i}, x_{j}\right)} \# \widehat{\mathcal{M}}(\phi) T^{\phi} x_{j}\right) \text {, }
$$


construct the corresponding matrix $\left(M_{i j}\right)=\left(\sum_{\phi \in \pi_{2}\left(x_{i}, x_{j}\right)} \# \widehat{\mathcal{M}}(\phi) T^{\phi}\right)$. Since $M_{i j} \in$ $\mathbb{Z}_{2}\left[H^{1}(Y ; \mathbb{Z})\right]$, it can be evaluated with respect to a given two-form $\eta$, producing a matrix $\left(M_{i j}(\eta)\right)=\left(\sum_{\phi \in \pi_{2}\left(x_{i}, x_{j}\right)} \# \widehat{\mathcal{M}}(\phi) T^{\eta(\phi)}\right) \in \operatorname{Mat}(\mathbb{A})$.

Take an arbitrary $k \times k$ minor of $M$, and compute its determinant. Denoting this function by $D$, the corresponding determinant of $M(\eta)$ is $D(\eta)$. As explained earlier, we want to find the likelihood for $D(\eta)=0$. For each pair of terms, we want to check

$$
\eta\left(\left(\phi_{1 \rho_{1}}+\cdots+\phi_{k \rho_{k}}\right)-\left(\phi_{1 \sigma_{1}}+\cdots+\phi_{k \sigma_{k}}\right)\right)=0 .
$$

Denote $\left(\phi_{1 \rho_{1}}+\cdots+\phi_{k \rho_{k}}\right)-\left(\phi_{1 \sigma_{1}}+\cdots+\phi_{k \sigma_{k}}\right)$ by $\phi$. There are two possibilities: either $\phi=0$ or $\phi \neq 0$. Note that $\phi_{i \rho_{i}}$ (resp. $\phi_{i \sigma_{i}}$ ) is a holomorphic disk connecting $x_{i}$ and $x_{\rho_{i}}$ (resp. $\left.\mathbf{x}_{\sigma_{i}}\right)$, so $\left(\phi_{1 \rho_{1}}+\cdots+\phi_{n \rho_{n}}\right)-\left(\phi_{1 \sigma_{1}}+\cdots+\phi_{n \sigma_{n}}\right)$ corresponds to a periodic domain in $\pi_{2}\left(x_{1}, x_{1}\right)$. Hence, by assumption, $\Omega(\phi)=0, \omega(\phi) \neq 0$ when $\phi \neq 0$, while $\eta(\phi)$ may or may not be zero.

In other words, when we write $D(\Omega)$ as a formal sum, all terms are identical. For $D(\omega)$, they cannot be identical unless they are initially identical in $D$. For a general $D(\eta)$, the bigger the kernel of $\eta$ is, the more terms in the summand are equal. Therefore, $D(\omega)=0$ implies $D(\eta)=0$ and $D(\eta)=0$ implies $D(\Omega)=0$; but the reverse is not true. Apply Lemma 2.2, we obtain part (3) of our proposition.

When $\phi \neq 0, \omega(\phi) \neq 0$, so $D(\omega)$ equals zero if and only if $D$ equals zero. This implies rank $M=\operatorname{rank} M(\omega)$, proving part (2).

Since all terms in $D(\Omega)$ are identical, we may replace all $T^{\phi}$ with 1 , and denote the corresponding matrix as $M(0)$. Then, $D(\Omega)=0$ if and only if $D(0)=0$, so rank $M(\Omega)=$ rank $M(0)$. Observe that $M(0)$ corresponds to the boundary map for the ordinary unperturbed $H F(Y)$, which proves part (1).

Similar results hold for $\mathrm{HF}^{+}$in a nontorsion $\operatorname{Spin}^{c}$ structure $\mathfrak{s}$ :

Proposition 2.3 (1) When $\mathfrak{s}$ is a nontorsion $\operatorname{Spin}^{c}$ structure, $\mathrm{HF}^{+}(Y, s ; \eta)$ is finitely generated, and the Euler characteristic

$$
\chi\left(H F^{+}(Y, \mathfrak{s} ; \eta)\right)=\chi\left(H F^{+}(Y, s)\right)= \pm \tau_{t}(Y, \mathfrak{s}),
$$

where $\tau_{t}$ is Turaev's torsion function, with respect to the component $t$ of $H^{2}(Y ; \mathbb{R})-0$ containing $c_{1}(\mathfrak{s})$.

(2) The rank of $\mathrm{HF}^{+}(Y, \mathfrak{s} ; \Omega)$ over $\mathbb{A}$ is equal to the rank of the ordinary unperturbed $H F^{+}(Y, \mathfrak{s})$ over $\mathbb{Z}_{2}$.

(3) The rank of $\mathrm{HF}^{+}(Y ; \omega)$ over $\mathbb{A}$ is equal to the rank of the nontorsion part of the completely twisted $\underline{H F^{+}}\left(Y ; \mathbb{Z}_{2}\left[H^{1}(Y ; \mathbb{Z})\right]\right)$ over the ring $\mathbb{Z}\left[H^{1}(Y ; \mathbb{Z})\right]$. 
(4) In general, as $\mathbb{A}$-vector spaces, we have the inequality

$$
\operatorname{rank} H F^{+}(Y, \mathfrak{s} ; \omega) \leq \operatorname{rank} H F^{+}(Y, \mathfrak{s} ; \eta) \leq \operatorname{rank} H F^{+}(Y, \mathfrak{s} ; \Omega) .
$$

Proof The first part is proved by a similar argument as in Ozsváth and Szabó [4, Section 5]. As soon as we know that $\mathrm{HF}^{+}(Y, s ; \eta)$ is finitely generated, the argument in the proof of Proposition 2.1 can be adopted to prove the remaining parts.

\section{Computations for $T^{3}$}

In this section, we compute the perturbed Heegaard Floer homology for $T^{3}$. It was shown in Ozsváth and Szabó $\left[2\right.$, Section 8.4] that $\widehat{H F}\left(T^{3}\right) \cong H^{2}\left(T^{3} ; \mathbb{Z}\right) \oplus H^{1}\left(T^{3} ; \mathbb{Z}\right)$. By Proposition 2.1 , this is equivalent to $\widehat{H F}\left(T^{3} ; \Omega\right)=\mathbb{A}^{6}$. We aim to compute $\widehat{H F}\left(T^{3} ; \eta\right)$ for a general $\eta \in H^{2}\left(T^{3} ; \mathbb{Z}\right)=\mathbb{Z}^{3}$. Our result is:

Theorem 3.1 For a nonzero two-form $\eta, \widehat{H F}\left(T^{3} ; \eta\right)=\mathbb{A}^{2}$.

Our proof is based on a certain "special Heegaard diagram" first introduced by Ozsváth and Szabó [6], in which some genus $2 g+1$ Heegaard diagrams were constructed for $\Sigma_{g}$ bundle over $S^{1}$. In this paper, we use a slightly different presentation by drawing two standard $4 g$-gons to represent left and right hand side genus- $g$ surfaces respectively. Two holes are created in either side to form a connected sum of a $2 g+1$ Heegaard surface.
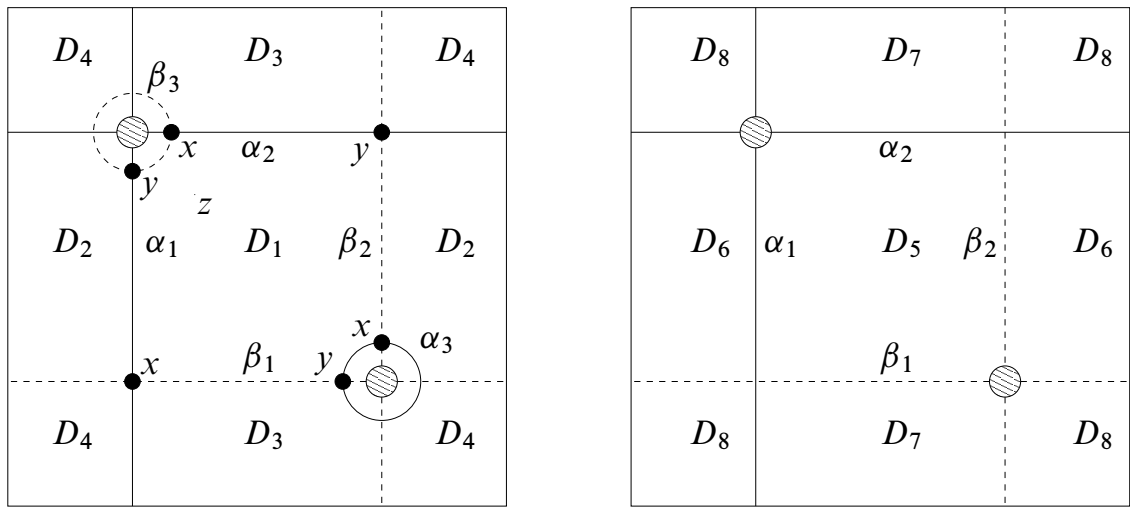

Figure 1: This is a Heegaard diagram for $T^{3}$ : Tori are represented by rectangles with opposite sides identified, and two holes are punctured in each side, represented by shaded disks. The Heegaard surface is divided into eight regions $D_{1}, \ldots, D_{8}$ by $\alpha$ 's and $\beta$ 's. 
Figure 1 is a special diagram for $T^{3}$ : rectangles with opposite sides are identified to represent tori. The curves $\alpha$ and $\beta$ are drawn on both sides and connected through the holes to represent closed curves. Put the base point $z$ in the region $D_{1}$. Note that this is NOT an admissible diagram as periodic domains $\mathcal{D}_{1}:=D_{2}+D_{4}+D_{6}+D_{8}$, $\mathcal{D}_{2}:=D_{3}+D_{4}+D_{7}+D_{8}$ and $\mathcal{D}_{3}:=D_{5}+D_{6}+D_{7}+D_{8}$ have positive coefficients only.

Nevertheless, Figure 1 is useful in the computation of the perturbed Floer Homology $\widehat{H F}\left(T^{3} ; \eta\right)$; the only restriction of nonadmissibility is given by $\eta\left(\mathcal{D}_{i}\right)>0$ for all $i$. However, nonadmissible diagrams at least can still be used to compute $\widehat{H F}\left(T^{3} ; \omega\right)$.

Lemma 3.2 For a generic two-form $\omega, \widehat{H F}\left(T^{3} ; \omega\right)=\mathbb{A}^{2}$.

Proof The Adjunction Inequality in [4, Section 7] implies $\widehat{H F}\left(T^{3}, \mathfrak{s} ; \omega\right)$ vanishes for any nontorsion $\operatorname{Spin}^{c}$ structures $\mathfrak{s}$. Recall the first Chern class formula [4, Section 7.1]

$$
\left\langle c_{1}\left(\mathfrak{s}_{y}\right),[\mathcal{P}]\right\rangle=\chi(\mathcal{P})-2 \bar{n}_{z}(\mathcal{P})+2 \sum_{p \in y} \bar{n}_{p}(\mathcal{P}),
$$

where $\mathfrak{s}_{y}$ is a $\operatorname{Spin}^{c}$ structure corresponding to $y$. We find two generators $x$ and $y$ in $\widehat{C F}\left(T^{3}, \mathfrak{s}_{0} ; \omega\right)$, where $\mathfrak{s}_{0}$ is the unique torsion $\operatorname{Spin}^{c}$ structure of $T^{3}$.

Observe that $D_{1}$ is a holomorphic disk connecting $x$ to $y$. Any other holomorphic disks $\phi$ connecting $x$ to $y$ must differ from $D_{1}$ by a periodic domain with Maslov index 0 , hence $\phi$ can be written as $D_{1}+k_{1} \mathcal{D}_{1}+k_{2} \mathcal{D}_{2}+k_{3} \mathcal{D}_{3}$ for some integers $k_{1}, k_{2}$ and $k_{3}$. A holomorphic disk has a nonnegative coefficient in each region, in particular $D_{2}, D_{3}$ and $D_{5}$. Hence $k_{i} \geq 0$, which implies that $\phi$ strictly contains $D_{1}$.

We claim that there is no holomorphic disk connecting $y$ to $x$. Otherwise, suppose $\psi$ is a disk connecting $y$ to $x$ with the smallest area. Then

$$
\begin{aligned}
\left(\partial^{+}\right)^{2}[x, i] & =\left(\partial^{+}\right)\left(T^{\omega\left(D_{1}\right)}[y, i-1]+\cdots\right) \\
& =T^{\omega\left(D_{1}\right)} \cdot T^{\omega(\psi)}\left[x, i-1-n_{z}(\psi)\right]+\text { higher order terms in } T,
\end{aligned}
$$

contradicting $\left(\partial^{+}\right)^{2}=0$.

Hence, $\partial y=0$, and for any holomorphic disk $\phi$ connecting $x$ to $y$, we have $n_{z}(\phi) \neq 0$. So $\partial x=0$, and consequently $\widehat{H F}\left(T^{3} ; \omega\right)=\mathbb{A}^{2}$.

Certain modifications of Figure 1 enable us to compute the perturbed Floer homology for some other two-form $\eta$. For example, Figure 2 can be used for $\eta_{1}$ with $\eta_{1}\left(\mathcal{D}_{1}\right)=$ $\eta_{1}\left(\mathcal{D}_{2}\right)=0$ and Figure 3 can be used for $\eta_{2}$ with $\eta_{2}\left(\mathcal{D}_{1}\right)=\eta_{2}\left(\mathcal{D}_{3}\right)=0$. In both 

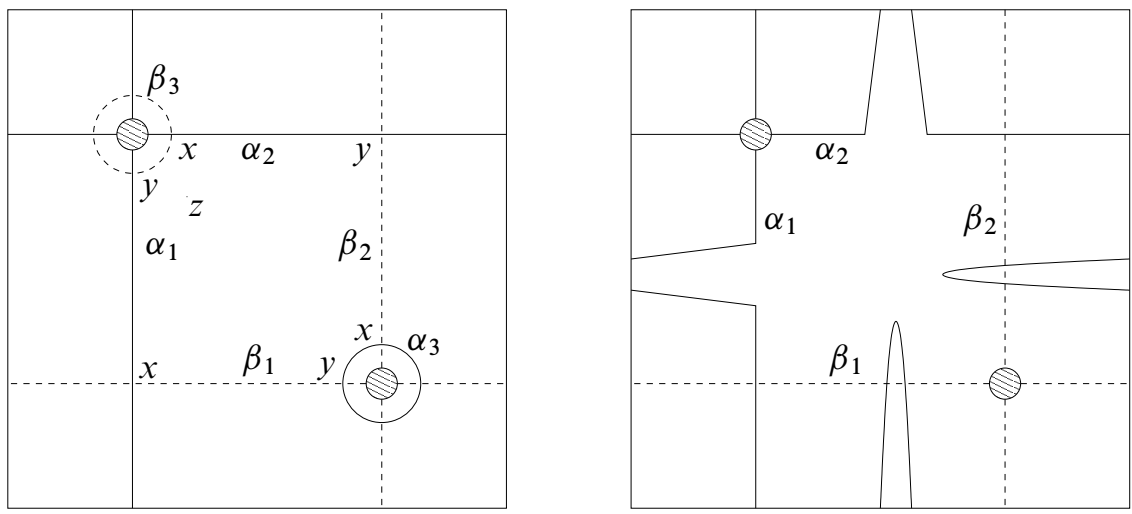

Figure 2: This is a modified Heegaard diagram for $T^{3}: \alpha_{1}$ and $\alpha_{2}$ are twisted across $\beta_{2}$ and $\beta_{1}$ respectively. In this diagram, there exists a twoform $\eta_{1}$ such that $\eta_{1}\left(\mathcal{D}_{1}\right)=\eta_{1}\left(\mathcal{D}_{2}\right)=0$.
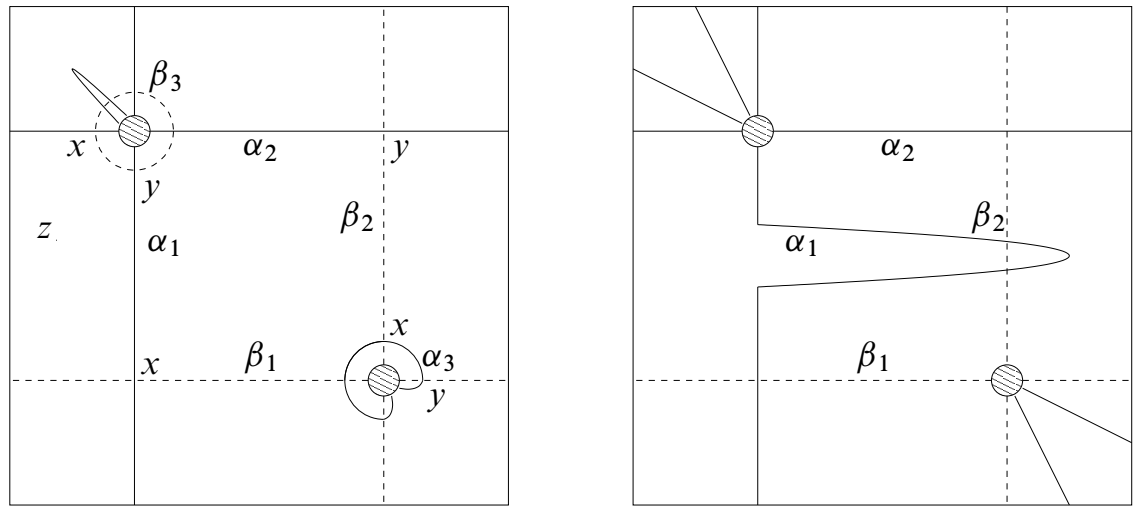

Figure 3: This is a modified Heegaard diagram for $T^{3}: \alpha_{1}$ is twisted across $\beta_{2}$, and $\alpha_{3}$ is winding across $\beta_{3}$. In this diagram, there exists a two-form $\eta_{2}$ such that $\eta_{2}\left(\mathcal{D}_{1}\right)=\eta_{1}\left(\mathcal{D}_{3}\right)=0$.

cases, there are two generators $x$ and $y$, and no boundary map by a similar argument. Hence, $\widehat{H F}\left(Y ; \eta_{1}\right)=\widehat{H F}\left(Y ; \eta_{2}\right)=\mathbb{A}^{2}$.

Figure 4 is another Heegaard diagram for $T^{3}$, and it is admissible. Unlike previous cases though, this time we have six generators, labeled by $x, y, p, p^{\prime}, q$ and $q^{\prime}$, which is reasonable since $\widehat{H F}\left(T^{3}\right)$ has rank six. The boundary map in our case is complicated as well: Figure 4 can be used for computing $\widehat{H F}\left(T^{3}, \Omega\right), \widehat{H F}\left(T^{3}, \eta_{1}\right)$, $\widehat{H F}\left(T^{3}, \eta_{2}\right)$ and $\widehat{H F}\left(T^{3}, \omega\right)$, and the answers are $\mathbb{A}^{6}$ for the first three homologies, and $\mathbb{A}^{2}$ for the last homology. So there must exist some cancelling pair of holomorphic 
disks for the area form $\Omega$ that is no longer cancellable in $\eta_{1}, \eta_{2}$ or $\omega$. It would be helpful if all boundary maps could be found explicitly.
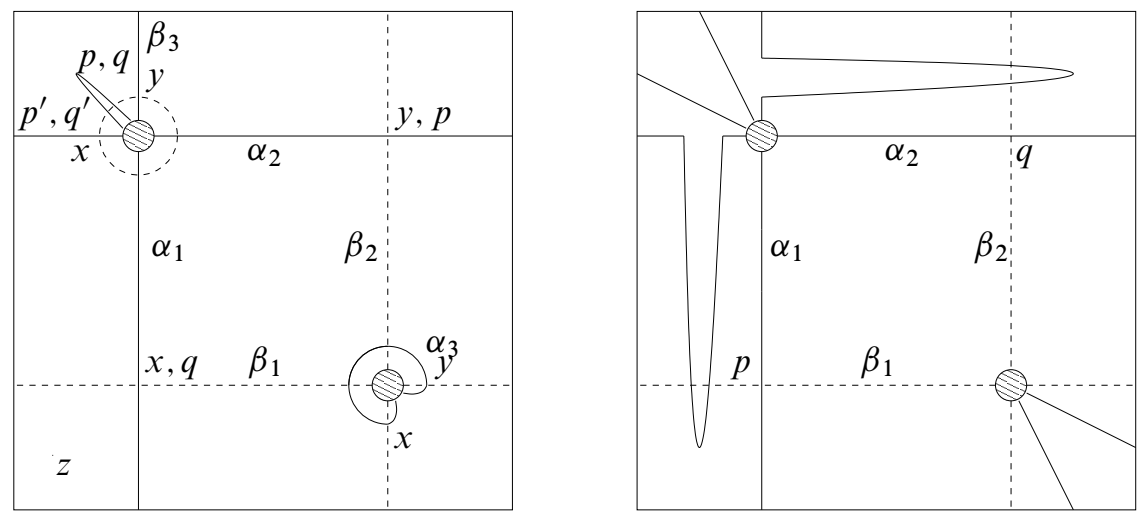

Figure 4: This is an admissible diagram for $T^{3}: \alpha_{1}, \alpha_{2}$ are twisted across $\beta_{2}, \beta_{1}$, respectively, and $\alpha_{3}$ is winding across $\beta_{3}$. In this diagram, there exists a two-form $\eta$ such that $\eta\left(\mathcal{D}_{1}\right)=\eta\left(\mathcal{D}_{2}\right)=\eta\left(\mathcal{D}_{3}\right)=0$.

Now, we are prepared for the proof of Theorem 3.1. The idea is to start from some special two-form $\eta^{\prime}$ such that $\widehat{H F}\left(T^{3} ; \eta^{\prime}\right)=\mathbb{A}^{2}$ and $\operatorname{Ker}\left(\eta^{\prime}\right)$ is a codimension-1 subspace of $H^{2}\left(T^{3} ; \mathbb{Q}\right)=\mathbb{Q}^{3}$ (both $\eta_{1}$ and $\eta_{2}$ meet the requirements). Then we look for some element of the large automorphism group of $T^{3}$ to map $\operatorname{Ker}\left(\eta^{\prime}\right)$ to some given hyperplane of $\mathbb{Q}^{3}$, namely $\operatorname{Ker}(\eta)$. Functoriality of Heegaard Floer homology implies that the corresponding map from $\widehat{H F}\left(T^{3} ; \eta^{\prime}\right)$ to $\widehat{H F}\left(T^{3} ; \eta\right)$ is also an isomorphism, giving $\mathbb{A}^{2}$.

Proof of Theorem 3.1 As mentioned earlier, both $\eta_{1}$ and $\eta_{2}$ can serve as our $\eta^{\prime}$. Instead, we describe a nonconstructive way of finding $\eta^{\prime}$ that is valid in general situation. Fix an admissible Heegaard diagram and find all generators and boundary maps. There are only finitely many $\phi$ 's as proven by Proposition 2.1, so we can find a hyperplane $H^{\prime}$ in $\mathbb{Q}^{3}$ missing all the $\phi$ 's. Let $\eta^{\prime}$ evaluate zero on the hyperplane, and nonzero elsewhere. Clearly, $\operatorname{Ker}\left(\eta^{\prime}\right)=H^{\prime}$ has codimension 1 . Since $\eta^{\prime}$ evaluates nonzero on all $\phi$ 's, it essentially plays the role of a generic form $\omega$, hence by Lemma 3.2, $\widehat{H F}\left(T^{3} ; \eta^{\prime}\right)=\widehat{H F}\left(T^{3} ; \omega\right)=\mathbb{A}^{2}$.

Suppose $\operatorname{Ker}(\eta)$ is another hyperplane $H$. It is always possible to find some element of $\mathrm{SL}_{3}(\mathbb{Z})$ that maps $H^{\prime}$ to $H$. On the other hand, any element of $\mathrm{SL}_{3}(\mathbb{Z})$ can be realized as the underlying $H^{2}\left(T^{3} ; \mathbb{Z}\right)$ map induced by some $T^{3}$ automorphism, say $\Phi$ in this case. Then, $\widehat{H F}\left(T^{3} ; \eta\right)=\widehat{H F}\left(T^{3} ; \Phi^{*}(\eta)\right)=\widehat{H F}\left(T^{3} ; \eta^{\prime}\right)=\mathbb{A}^{2}$. 


\section{Computations for $\Sigma_{g} \times S^{1}$}

In this section, we compute the perturbed Heegaard Floer homology of $\Sigma_{g} \times S^{1}$ for $g>1$. Our result is:

Theorem 4.1 For a nonzero two-form $\eta$,

$$
\left.H F^{+}\left(\Sigma_{g} \times S^{1}, k ; \eta\right)=(\mathbb{A}[U] / U)^{(2 g-2}\right),
$$

where $d=g-1-|k|, k \neq 0$.

Here, $H F^{+}\left(\Sigma_{g} \times S^{1}, k ; \eta\right)$ denotes the summand of $H F^{+}\left(\Sigma_{g} \times S^{1} ; \eta\right)$ corresponding to the $\operatorname{Spin}^{c}$ structure $\mathfrak{s}$ with $\left\langle c_{1}(\mathfrak{s}),\left[\Sigma_{g}\right]\right\rangle=2 k$ and $\left\langle c_{1}(\mathfrak{s}), \gamma \times S^{1}\right\rangle=0$ for all curves $\gamma \subset \Sigma_{g}$.

Remark 4.2 When $k \neq 0$, ie $\left\langle c_{1}(\mathfrak{s}),\left[\Sigma_{g}\right]\right\rangle \neq 0$, perturbations in the $\Sigma_{g}$-direction do not have any effect on the Heegaard Floer homology. Hence, we can restrict our consideration of $\eta$ to the subspace $H^{2}\left(\Sigma_{g} ; \mathbb{Z}\right)$ of $H^{1}\left(\Sigma_{g} \times S^{1} ; \mathbb{Z}\right)$.

We can compare this result with the unperturbed case computed by Ozsvath and Szabó in $[3$, Section 9]:

Theorem 4.3 Fix an integer $k \neq 0$. Then, there is an identification of $\mathbb{Z}$-modules

$$
H F^{+}\left(\Sigma_{g} \times S^{1}, k\right) \cong X(g, d),
$$

where $d=g-1-|k|$, and

$$
X(g, d)=\bigoplus_{i=0}^{d} \Lambda^{2 g-i} H^{1}\left(\Sigma_{g}\right) \otimes_{\mathbb{Z}}\left(\mathbb{Z}[U] / U^{d-i+1}\right) .
$$

It is interesting to compare the Euler characteristic of $\mathrm{HF}^{+}$. Recall the following combinatorial identity:

Lemma 4.4 $\sum_{i=1}^{m}(-1)^{i+1} i\left(\begin{array}{c}2 g \\ m-i\end{array}\right)=\left(\begin{array}{c}2 g-2 \\ m-1\end{array}\right)$.

Proof Write out the identity $\left(x /(1+x)^{2}\right)(1+x)^{2 g}=x(1+x)^{2 g-2}$ in formal series

$$
\left(\sum_{i=0}^{\infty}(-1)^{i+1} i x^{i}\right) \cdot\left(\sum_{i=0}^{\infty}\left(\begin{array}{c}
2 g \\
m-i
\end{array}\right) x^{m-i}\right)=\sum_{m=0}^{\infty}\left(\begin{array}{c}
2 g-2 \\
m-1
\end{array}\right) x^{m},
$$

and compare their coefficients for $x^{m}$. 
Hence, replacing $d$ by $m-1$ in the formula, we have

$$
\chi\left(H F^{+}\left(\Sigma_{g} \times S^{1}, k\right)\right)=\sum_{i=0}^{d}(-1)^{i+1}(d-i+1)\left(\begin{array}{c}
2 g \\
i
\end{array}\right)=(-1)^{d-1}\left(\begin{array}{c}
2 g-2 \\
d
\end{array}\right) .
$$

This agrees with the Euler characteristic of $\mathrm{HF}^{+}\left(\Sigma_{g} \times S^{1}, k ; \eta\right)$ as expected from Proposition 2.3. Compare also with Jabuka and Mark [1, Corollary 1.8] and Salamon [7, Section 6].

In fact, we will use the Euler characteristic as one of the key ingredients in our proof of Theorem 4.1.

Just like the case of $T^{3}$, we divide the proof of Theorem 4.1 into two steps:

Step 1 We use a special Heegaard diagram for $\Sigma_{g} \times S^{1}$ in Figure 5. There are two generators in $\operatorname{spin}^{c}$ structures $k=g-1$, marked out in the figure by dots and squares. In general, there are $2\left(\begin{array}{c}2 g-1 \\ d\end{array}\right)$ generators in $\operatorname{Spin}^{c}$ structure $k=g-1-d$, obtained by moving $d$ of the intersection points between $\alpha_{i}$ and $\beta_{i}(i \leq 2 g)$ from the upper polygon to the lower polygon. These generators are further divided into four classes:

- Class A consists of $\left(\begin{array}{c}2 g-2 \\ d-1\end{array}\right)$ generators. These generators have the intersection between $\alpha_{2 g-1}$ and $\beta_{2 g-1}$ in the lower polygon.

- Class A' consists of $\left(\begin{array}{c}2 g-2 \\ d-1\end{array}\right)$ generators. These generators have the intersection between $\alpha_{2 g}$ and $\beta_{2 g}$ in the lower polygon.

- Class $B$ consists of $\left(\begin{array}{c}2 g-2 \\ d\end{array}\right)$ generators. These generators have the intersection between $\alpha_{2 g}$ and $\beta_{2 g}$ in the upper polygon.

- Class $B$ ' consists of $\left(\begin{array}{c}2 g-2 \\ d\end{array}\right)$ generators. These generators have the intersection between $\alpha_{2 g-1}$ and $\beta_{2 g-1}$ in the upper polygon.

Denote the hexagon region where we put the base point $z$ by $D$ and the corresponding hexagon region in the lower polygon by $D^{\prime}$. Pairs of generators from Class A to A' are connected by $D^{\prime}$, while pairs of generators from Class B to B' are connected by $D$.

All of the information gathered so far for the chain complex $\widehat{C F}^{+}$may be summarized in Figure 6. If there were no other holomorphic disks besides $D$ and $D^{\prime}$ in the diagram, then

$$
H F^{+}=(\mathbb{A}[U] / U)^{\left(\begin{array}{c}
2 g-2 \\
d
\end{array}\right)} .
$$

However, with a little assumption on the two-form $\omega$, we would be able to prove the fact without much further knowledge of the boundary map $\partial$. 

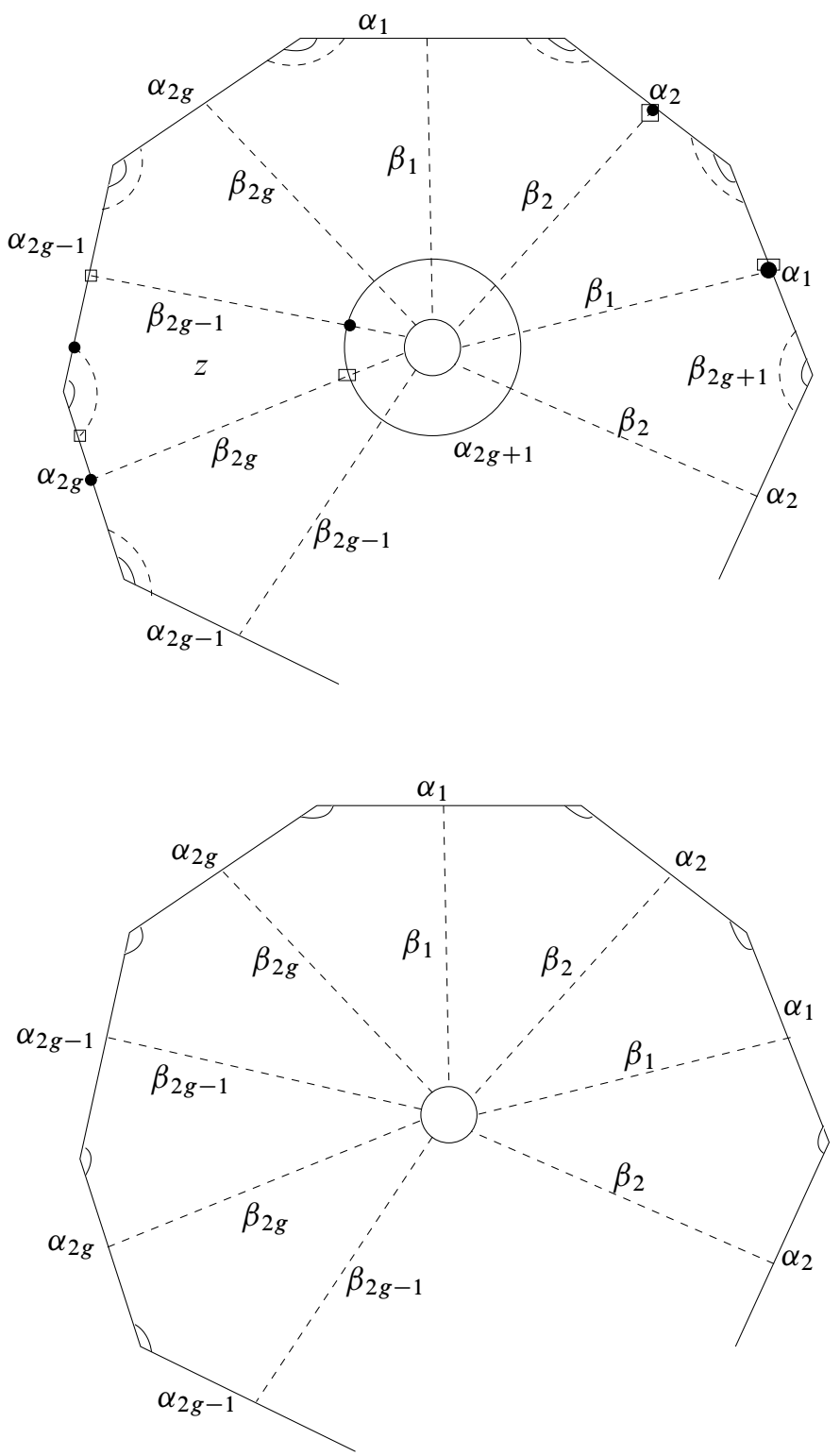

Figure 5: This is a nonadmissible Heegaard diagram for $\Sigma_{g} \times S^{1}$. Two holes are punctured in each $4 g$-gons and connected to a genus $2 g+1$ Heegaard surface. The two generators in $\operatorname{spin}^{c}$ structures $k=2 g-2$ are marked out by dots and squares. In general, there are $2\left(\begin{array}{c}2 g-1 \\ d\end{array}\right)$ generators in $\operatorname{Spin}^{c}$ structure $k$, which are obtained by moving $d$ of the intersection points between $\alpha_{i}$ and $\beta_{i}$ from the upper polygon to the lower polygon. 

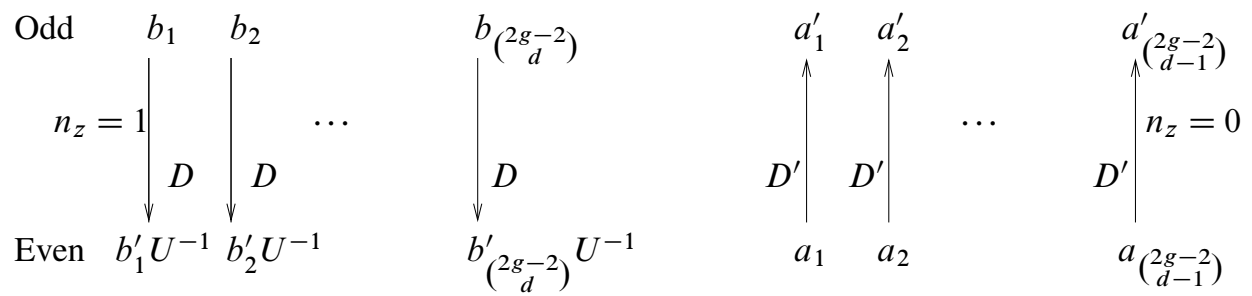

Figure 6: This diagram includes all the information we know about $\mathrm{CF}^{+}$. Class A, A', B and B' generators are denoted by $a_{1}, \ldots, a_{\left(\begin{array}{c}2 g-2 \\ d-1\end{array}\right)}, a_{1}^{\prime}, \ldots, a_{\left(\begin{array}{c}2 g-2 \\ d-1\end{array}\right)}^{\prime}, b_{1}, \ldots, b_{\left(\begin{array}{c}2 g-2 \\ d\end{array}\right)}$ and $b_{1}^{\prime}, \ldots, b_{\left(\begin{array}{c}2 g-2 \\ d\end{array}\right)}^{\prime}$,

respectively. In $\mathbb{Z} / 2 \mathbb{Z}$ grading, Classes $B$ and $A^{\prime}$ have odd degrees, and Classes A and B' have even degrees. $\mathrm{HF}^{+}$may be determined completely with this little amount of information.

Proposition 4.5 For a generic two-form $\omega$ with $\omega(D)=\omega\left(D^{\prime}\right) \ll \omega$ (other regions), we have

$$
\left.H F^{+}\left(\Sigma_{g} \times S^{1}, k ; \omega\right)=(\mathbb{A}[U] / U)^{(2 g-2}{ }_{d}\right),
$$

where $d=g-1-|k|, k \neq 0$.

Proof Use $A, A^{\prime}, B$ and $B^{\prime}$ to denote the vector spaces generated by Class A, A', B and $\mathrm{B}^{\prime}$ generators respectively, and define

$$
\begin{aligned}
\text { Odd } & :=\left(B+A^{\prime}\right) \cdot\left(1+U^{-1}+U^{-2}+\cdots\right), \\
\text { Even } & :=\left(A+B^{\prime}\right) \cdot\left(1+U^{-1}+U^{-2}+\cdots\right), \\
M & :=B+A^{\prime} \cdot\left(1+U^{-1}+U^{-2}+\cdots\right), \\
N & :=B \cdot\left(U^{-1}+U^{-2}+\cdots\right), \\
\operatorname{Ker} & :=\text { Kernel of the boundary map Odd } \longrightarrow \text { Even, } \\
\widetilde{I m} & :=\text { Image of the boundary map Even } \longrightarrow \text { Odd, } \\
\widetilde{\mathrm{Ker}} & :=\text { projection of Ker into } M . \\
\widetilde{\mathrm{Im}} & :=\text { projection of Im into } M .
\end{aligned}
$$

We have the following:

- $\operatorname{Odd}=M \oplus N, \operatorname{Im} \subset \operatorname{Ker} \subset$ Odd

- $\operatorname{Ker} \cap N=0$.

Write elements of $N$ in the most general form $x=\sum b_{i} U^{-j} k_{i j}$, where $k_{i j} \in \mathbb{A}$. Suppose $k_{i_{1} j_{1}}$ is one of the coefficients with the lowest order term in $T$. Then $\partial x=b_{i_{1}}^{\prime} U^{-\left(j_{1}-1\right)} \cdot\left(k_{i_{1} j_{1}} T^{\omega(D)}+\right.$ higher order terms in $\left.\mathrm{T}\right)+\cdots$. 
But $\partial x=0$ if $x \in$ Ker, which is not possible unless $x=0$.

Hence, all information of Ker is contained within $\widetilde{\mathrm{Ker}}$, so we can restrict our attention to $\widetilde{\mathrm{Ker}}$; the same applies for Im and $\widetilde{\mathrm{Im}}$.

- $\widetilde{\mathrm{Im}}+B=M \supset \widetilde{\mathrm{Ker}}$.

Compute the determinant of the $\left(\begin{array}{c}2 g-2 \\ d-1\end{array}\right) \times\left(\begin{array}{c}2 g-2 \\ d-1\end{array}\right) \partial$-matrix from $A$ to $A^{\prime}$. There is a unique lowest order term $T$ in the determinant with order $\left(\begin{array}{c}2 g-2 \\ d-1\end{array}\right) \cdot \omega\left(D^{\prime}\right)$, hence it is nonzero, and so the map is surjective. The same argument carries on for larger spaces $A\left(1+U^{-1}+\cdots+U^{-k}\right)$, and the map is surjective onto $A^{\prime}\left(1+U^{-1}+\cdots+U^{-k}\right)$. Letting $k \rightarrow \infty$, we have proved $\widetilde{\mathrm{Im}}+B=M \supset \widetilde{\mathrm{Ker}}$.

- Therefore, $\operatorname{rank}\left(H F_{\text {odd }}^{+}\right) \leq \operatorname{rank} B=\left(\begin{array}{c}2 g-2 \\ d\end{array}\right)$. But $\chi\left(H F^{+}\right)=\left(\begin{array}{c}2 g-2 \\ d\end{array}\right)$, so we must have

$$
\operatorname{rank}\left(H F_{\text {odd }}^{+}\right)=\left(\begin{array}{c}
2 g-2 \\
d
\end{array}\right) \text { and } \operatorname{rank}\left(H F_{\text {even }}^{+}\right)=0 .
$$

- As shown above, we can choose a set of generators $x_{i} \in B \oplus N, i=1, \ldots,\left(\begin{array}{c}2 g-2 \\ d\end{array}\right)$, for $H F^{+}$. We want to prove $x_{i}$ in fact lies in $B$. This would imply $x_{i} \cdot U=0$, finishing the proof that $H F^{+}\left(\Sigma_{g} \times S^{1}, k ; \omega\right)=(\mathbb{A}[U] / U)^{(2 g-2} d{ }_{d}$.

Up to this point, we have not used any information from the boundary map in this special Heegaard diagram. Here is the place we have to use a little: upon investigating Figure 5, writing out all $k$-renormalizable periodic domain and finding out all possible topological disks with Maslov index 1, we find that no holomorphic disk connects generators from Class B to B' with $n_{z}=0$. In other words, when the boundary map $\partial$ is restricted to $B$ and $B^{\prime}$, it becomes zero. Write $x_{i}=\widetilde{x_{i}}+y_{i}$, where $\widetilde{x_{i}} \in B$ and $y_{i} \in N$. Then

$$
0=\partial\left(x_{i}\right)=\partial\left(\widetilde{x_{i}}\right)+\partial\left(y_{i}\right)=\partial\left(y_{i}\right) .
$$

But we know $\operatorname{Ker} \cap N=0$, so $y_{i}=0$.

Step 2 Since $\Sigma_{g}$ has a large symmetric group, the perturbed floer homology group is in some sense not sensitive to the exact direction of perturbations. More precisely:

Lemma 4.6 For any nonzero $\eta \in H^{1}\left(\Sigma_{g} ; \mathbb{Z}\right)$, we have $H F^{+}\left(\Sigma_{g} \times S^{1}, k ; \eta\right)=$ $H F^{+}\left(\Sigma_{g} \times S^{1}, k ; \omega\right)$ and $\widehat{H F}\left(\Sigma_{g} \times S^{1}, k ; \eta\right)=\widehat{H F}\left(\Sigma_{g} \times S^{1}, k ; \omega\right)$ as $\mathbb{A}$-vector spaces, for $k \neq 0$.

Proof The proof is parallel to that of $T^{3}$ : Find a special two-form $\eta^{\prime}$ such that $H F^{\circ}\left(\Sigma_{g} \times S^{1} ; \eta^{\prime}\right)=H F^{\circ}\left(\Sigma_{g} \times S^{1} ; \omega\right)$ and $\operatorname{Ker}\left(\eta^{\prime}\right)$ is a hyperplane $H^{\prime}$ of $H^{1}\left(\Sigma_{g} ; \mathbb{Q}\right)$. Supposing the kernel of $\eta$ is another hyperplane $H$, it is possible to find some element in $\operatorname{Sp}(2 g ; \mathbb{Z})$ that maps $H$ to $H^{\prime}$. On the other hand, a standard result on mapping class groups implies that any element in $\operatorname{Sp}(2 g ; \mathbb{Z})$ is induced by some elements of the mapping class group $\operatorname{Mod}_{g}$. Functoriality of $H F^{\circ}$ finishes the proof. 
Proof of Theorem 4.1 Applying Lemma 4.6 and Proposition 4.5, we have as $\mathbb{A}$-vector spaces

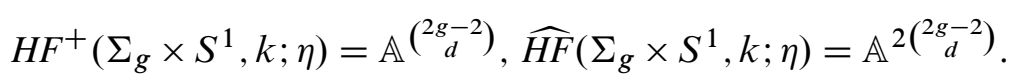

On the other hand, as an $A[U]-$ module, $H F^{+}\left(\Sigma_{g} \times S^{1}, k ; \eta\right)$ must have the general form $\mathbb{A}[U] / U^{k_{1}} \oplus \cdots \oplus \mathbb{A}[U] / U^{k_{n}}$. So by consideration of rank, we must have

$$
\left.H F^{+}\left(\Sigma_{g} \times S^{1}, k ; \eta\right)=(\mathbb{A}[U] / U)^{(2 g-2}\right) .
$$

Remark 4.7 For the case of the torsion $\operatorname{spin}^{c}$-structure $\mathfrak{s}_{0}$, our method suggests the conjecture that

$$
\left.H F^{+}\left(\Sigma_{g} \times S^{1}, \mathfrak{s}_{0} ; \omega\right)\right)=\mathbb{A}\left(\begin{array}{c}
2 g-2 \\
g-1
\end{array}\right) .
$$

But we need to first establish an analog of Proposition 2.3(1).

\section{References}

[1] S Jabuka, T E Mark, Product formulae for Ozsváth-Szabó 4-manifold invariants, Geom. Topol. 12 (2008) 1557-1651 MR2421135

[2] P Ozsváth, Z Szabó, Absolutely graded Floer homologies and intersection forms for four-manifolds with boundary, Adv. Math. 173 (2003) 179-261 MR1957829

[3] P Ozsváth, Z Szabó, Holomorphic disks and knot invariants, Adv. Math. 186 (2004) 58-116 MR2065507

[4] P Ozsváth, Z Szabó, Holomorphic disks and three-manifold invariants: properties and applications, Ann. of Math. (2) 159 (2004) 1159-1245 MR2113020

[5] P Ozsváth, Z Szabó, Holomorphic disks and topological invariants for closed threemanifolds, Ann. of Math. (2) 159 (2004) 1027-1158 MR2113019

[6] P Ozsváth, Z Szabó, Heegaard Floer homology and contact structures, Duke Math. J. 129 (2005) 39-61 MR2153455

[7] D A Salamon, Seiberg-Witten invariants of mapping tori, symplectic fixed points, and Lefschetz numbers, from: "Proceedings of 6th Gökova Geometry-Topology Conference", Turkish J. Math. 23 (1999) 117-143 MR1701642

[8] $\mathbf{Z ~ W u}$, Perturbed Floer homology of some fibered three-manifolds, in preparation

Department of Mathematics, Princeton University

Princeton, NJ 08544-1000, USA

zhongtao@math.princeton.edu

Received: 10 October 2008 Revised: 23 January 2009 